\title{
Diferencias territoriales en las percepciones políticas en Chile. Una aproximación operacional a la escala nacional y regional ${ }^{\star}$
}

\author{
Territorial differences in political perceptions in Chile. \\ An operational approach to the national and regional level
}

\author{
Gonzalo Delamaza** \\ Luis Eduardo Thayer Correa*** \\ José Manuel Gaete ${ }^{* * *}$
}

Centro de Investigación Sociedad y Políticas Públicas, Universidad de Los Lagos, Santiago, Chile

Recibido: 21 de enero de 2015. Aprobado: 25 de mayo de 2015

\section{Resumen}

La investigación que se presenta tiene por objetivo visibilizar algunas diferencias significativas en distintas escalas territoriales, en cuanto a percepciones políticas relevantes para la gobernanza democrática y la constitución de la esfera pública, como adhesión a la democracia y confianza interpersonal y hacia las instituciones, entre otros. Para ello se comparan resultados de encuestas nacionales con datos del Barómetro Regional 2013, una encuesta aplicada a muestras representativas de las regiones de Los Ríos, Los Lagos y Bío Bío. El análisis muestra que las opiniones y percepciones regionalizadas difieren significativamente, no solo respecto del conjunto del país, sino también entre las mismas regiones. Con ello se deja en evidencia que uno de los problemas de la esfera pública nacional es que aquello que

Este artículo forma parte de los resultados de investigación del Proyecto Fondecyt Regular N 1120770.

* Sociólogo por la Universidad Católica, Doctor en Sociología por la Universidad de Leiden, Profesor Titular de la Universidad de Los Lagos. Director del Centro de Investigación Sociedad y Políticas Públicas en Santiago. Correo electrónico: gonzalo.delamaza@ulagos.cl

*** Sociólogo Universidad ARCIS, Doctor en Sociología por la Universidad Complutense de Madrid, Profesor Asociado de la Universidad de Los Lagos, Sub-director del Centro de Investigación Sociedad y Políticas Públicas en Santiago. Correo electrónico: luis.thayer@ulagos.cl

**** Sociólogo por la Universidad Católica, Doctor en Sociología por la Universidad de Salamanca, Investigador Postdoctoral Centro de Investigación Sociedad y Políticas Públicas en Santiago. Correo electrónico: jmgaete@ yahoo.com 
se presenta como el "nosotros" -la comunidad política- estaría escindido de las experiencias y las subjetividades que ese "nosotros" pretende representar. Y que la incorporación de la diferenciación regional permite un enfoque más preciso sobre la gobernanza democrática.

Palabras claves: percepciones políticas, subjetividad regional, encuestas.

\begin{abstract}
The research presented aims to make visible some significant differences in different territorial scales, in terms of political perceptions relevant to democratic governance and the constitution of the public sphere, as commitment to democracy and interpersonal trust and to institutions, among others. National survey results are compared with data from the Regional Barometer 2013, a survey of representative samples of the regions of Los Ríos, Los Lagos and Bío Bío. The analysis shows that regional opinions and perceptions differ significantly, not only with the whole country, but also between the regions themselves. This makes it clear that one of the problems of the national public sphere is that what is presented as the "we" -the political community- would be split from the experiences and subjectivities that "we" meant to represent. And that incorporating regional differentiation enables a more precise focus on democratic governance.
\end{abstract}

Keywords: political perceptions, regional subjectivity, surveys.

\title{
Introducción
}

¿Cuánto importa el territorio en la percepción política y social de sus habitantes? ¿Qué alcance tienen las mediciones aplicadas a muestras nacionales -los promedios- en relación a la percepción política, si se toma el punto de vista de las y los ciudadanos y su habitar en el territorio? La emergencia de conflictos regionales diferenciados en Chile en los últimos años ha vuelto a poner en agenda la heterogeneidad de las situaciones que se viven en los distintos territorios (regiones, comunas) a lo largo y ancho del país. Sin embargo, el grueso del conocimiento que se genera sobre percepciones políticas se basa en agregados nacionales que a su vez sirven de insumo para la toma de decisiones políticas precisamente en el ámbito nacional (gobierno central y partidos políticos). 
¿Cuánto sabemos, en cambio, sobre esas mismas variables en el ámbito de cada una de las regiones del país (para no referirnos a escalas aún más reducidas)? ¿Se diferencian o no las percepciones políticas según el lugar en que se habita? En este artículo se propone una comparación de percepciones políticas relevantes para la gobernanza democrática medidas a través de encuestas presenciales en base a muestras aleatorias entre dos escalas territoriales: el país y la región. La hipótesis que guía el trabajo es que aún en un Estado unitario y en un país relativamente homogéneo culturalmente, el territorio sí importa a la hora de percibir la democracia, las instituciones, la situación personal y del país. La contribución del trabajo consiste en aportar información empírica nueva para respaldar y dimensionar las magnitudes de este aserto, información que normalmente no está disponible en el debate nacional.

La verificación de dicha hipótesis tendría efectos tanto de conocimiento como relativos a la actividad política (incluyendo el diseño de políticas públicas). Si la escala territorial muestra variaciones significativas respecto de los agregados estadísticos del orden nacional, entonces el conocimiento de la subjetividad diferenciada por territorio se vuelve un importante campo de indagación que debe ser abordado para una mejor comprensión de la sociedad. Y, al mismo tiempo, el escaso conocimiento de las realidades subjetivas de las regiones y de otro tipo de territorios podría considerarse como una barrera importante para el surgimiento de proyectos descentralizadores y en general para la descentralización de la actividad política.

El artículo desarrolla un marco conceptual en torno a tres variables políticas: adhesión a la democracia, confianza y percepción de la situación personal y del país. Este marco tiene un sesgo marcadamente operacional, por una razón práctica: para los efectos de nuestra hipótesis nos interesa aquello que normalmente se mide a través de encuestas reconocidas como fuente de conocimiento válido en el país, principalmente las desarrolladas por el Programa de Naciones Unidas para el Desarrollo (PNUD). Luego se comparan datos nacionales recientes, surgidos de algunas de las encuestas más reconocidas $\mathrm{y}$ validadas, con las mismas preguntas aplicadas en una muestra representativa a nivel regional en tres regiones de Chile (Bío Bío, Los Ríos y Los Lagos) a través de la encuesta Barómetro Regional llevada a cabo en el marco del proyecto Fondecyt $\mathrm{N}^{\circ} 1120770^{1}$. Se introducen luego dos variables intervinientes que operan como diferenciadores políticos, usualmente considerados en el ámbito nacional (autoadscripción política y participación en organizaciones) para determinar si ellas operan del mismo modo en la esfera regional.

1 La encuesta ya había sido aplicada inicialmente en 2011 y validada en la ocasión. 
En la sección final se concluye respecto de la importancia de la escala territorial regional en relación a la percepción política en Chile en la actualidad.

\section{Subjetividad, pluralismo y territorio}

La vida política en una democracia, tal como señalara Lechner (1988), no puede concebirse a partir de una unidad nacional o pre-social que excluya las particularidades subjetivas que dan cuerpo a las diferencias. Con ello buscaba poner en relieve el principio de la pluralidad como un elemento constitutivo del espacio público (Arendt, 2003). En esta senda, el primer supuesto del que parte nuestro trabajo es que las particularidades subjetivas relativas a temas políticos y percepción de lo público que se expresan en los territorios y regiones, han tenido escasa presencia en la constitución del espacio público en Chile. Este fenómeno tiene raigambres históricas relativas a la constitución del Estado-nación, dada la preeminencia del Estado central tanto sobre la sociedad en general como sobre las regiones que desafiaron la hegemonía de la capital (Góngora, 1980; Boisier, 2010; Salazar y Pinto, 1999). Durante el siglo XX es la hegemonía del sistema de partidos políticos nacionales y su articulación con el Estado desarrollista, basado en un consenso de las clases involucradas en el modelo de desarrollo (Garretón, 1985), lo que inhibe la emergencia de una voz pública regional estable. Desde la recuperación de la democracia en 1990, dicho fenómeno se expresa nuevamente, a partir del "modelo de gobernabilidad" que definió la transición, impidiendo de diversas formas la pluralización del espacio público hacia los ámbitos regionales y locales (Delamaza, 2014; Navarrete, 2012; Joignant, 2012)2. Visto este problema desde el punto de vista del proceso institucional de descentralización, Angell, Lowden y Thorp (2001) argumentan en términos comparativos con América Latina, señalando el carácter excepcional de Chile, en cuanto la descentralización nunca habría estado ligada a la democratización y a la liberalización del país. Dicha afirmación podría considerarse válida al menos desde 1859, fecha del último levantamiento regional en contra del centralismo. Por su parte Falleti (2010) establece una teoría secuencial de la descentralización en la cual sus resultados dependen del orden en la que se implementan las

2 Boisier (2000) se refiere a la "vocación regionalista del régimen militar", dado que durante ese período se implementó una reforma que creó 13 regiones, atendiendo parcialmente a planteamientos de los técnicos desarrollistas de los años 70, entre los cuales se encontraba el mismo Boisier. Sin embargo, ello ocurrió como decisión político administrativa, sin ningún debate público y en condiciones de represión política y social. Más allá de las eventuales consecuencias descentralizadoras de dicha reforma, ella es una versión extrema del paradigma que prescinde del territorio como espacio construido y de la subjetividad como factor relevante. 
reformas, del actor que la origina y lidera (nacional o sub nacional) y de si la iniciativa descentralizadora se toma como reacción a un problema o crisis o bien si es solo el despliegue de una estrategia de los actores (self-reinforcing) (2010, p. 19). Aunque su estudio no incluye Chile, la tipología desarrollada indica que nuestro país aparecería como un caso de patrón nacional (liderazgo), acorde con la secuencia "descentralización administrativa - descentralización fiscal - descentralización política” y de tipo self reinforcing. Este es justamente el tipo en que menores impactos se observan en cuanto al surgimiento de polos regionales con mayor capacidad y proyección pública.

A partir de lo anterior nuestra investigación busca contribuir a mostrar algunas singularidades de la subjetividad regional y poner en evidencia uno de los problemas centrales de la arquitectura de la esfera pública nacional: que aquello que se presenta como el "nosotros" estaría escindido de las experiencias y las subjetividades que ese "nosotros" pretende representar.

El segundo supuesto que asumimos es que el centralismo es la fuerza responsable de separar el espacio público representado de los territorios vividos por los sujetos. Ello implica asumir que el centralismo es una dinámica presente no solo en el plano estructural e institucional, sino que está también asentado en la dimensión subjetiva. Es decir en el conjunto de percepciones, representaciones, creencias y opiniones con que los sujetos contribuyen a reproducirlo. A partir de esto planteamos que el desafío de largo plazo al que pretende contribuir nuestra investigación es el de visibilizar tanto las distinciones como las continuidades existentes entre las particularidades subjetivas de los territorios y regiones, en función de comprender la magnitud de dicha separación entre la experiencia en los territorios y la representación de lo público.

Desde el retorno de la democracia la pluralidad en la esfera política chilena ha estado demarcada principalmente por posiciones políticas e ideológicas que remiten en su origen a posiciones de clase de orden nacional. Esta ha sido asumida como una diferenciación que está presente de manera transversal en las regiones y territorios, estos no serían más que el contexto geográfico en que esa pluralidad se distribuye. Con excepción del conflicto entre el Estado chileno y las comunidades mapuche, el territorio ha estado al margen de la esfera política como factor de diferenciación y constitución de los sujetos. La consideración puramente formal del territorio, como la geografía en la que se distribuyen las posiciones partidarias nacionales, ha supuesto que se lo conciba exclusivamente desde una mirada formal e instrumental de cara al diseño de estrategias electorales y de distribución de cargos públicos. 
La representatividad electoral de los territorios no conlleva el que estos estén contenidos en la representación pública del "nosotros". El diseño institucional por su parte ha inhibido tanto el surgimiento de partidos regionales o regionalistas, que hacen política en nombre de las causas regionales (Navarrete, 2012), como de los incentivos para promover una dinámica política más autónoma de los representantes de las regiones (Joignant, 2012; Gaete, 2012). Otra consecuencia derivada de esta mirada territorialmente homogénea del espacio público, es la invisibilidad relativa a las diferencias regionales y territoriales, asumiéndose en el discurso público una homogeneidad no solo entre las regiones sino entre los territorios que las componen. Estas tendencias pueden cambiar un tanto en el futuro con la introducción de reformas electorales. Desde 2013 se eligen directamente los consejeros regionales y se ha aprobado una reforma del sistema electoral binominal que empezará a regir en 2017. En la medida que se elegirá mayor cantidad de representantes por distrito electoral -actualmente son solo dos- ello podría ofrecer nuevas oportunidades para una mayor diversificación de la representación, sin que todavía pueda saberse aún cuáles serán sus consecuencias concretas respecto del tema que comentamos.

El territorio ha comenzado a ser visto como un factor de diferenciación de sujetos en el espacio público a partir de los diversos conflictos regionales o territoriales y que han sido impulsados por actores del territorio y no por los actores políticos tradicionales. En los últimos años, varios conflictos socio territoriales alcanzaron gran repercusión pública y suscitaron diversas medidas de política pública. Algunos se relacionan con variables socio-ambientales (generación termoeléctrica y contaminación), megaproyectos energéticos y diversas manifestaciones masivas y protestas en localidades secundarias y alejadas de la capital (Punta Arenas, Aysén, Chiloé, Calama, Freirina, Petorca, Tocopilla, entre otros). Si nos remontamos unos años hacia atrás encontraremos diversos conflictos relacionados a problemas de territorios relativamente aislados o periféricos, la mayor parte de ellos asociados a consecuencias ambientales de proyectos de inversión y disputa por el uso de los recursos naturales en el territorio. Sin embargo no sólo se trata de conflictos ambientales, sino también de demandas sociales por la salud, el costo de vida, la participación en las decisiones locales o en general por que se provean servicios del Estado. Además, muchos de estos conflictos han sucedido en territorios indígenas o han tenido una importante participación de actores indígenas, en diversos territorios (Bengoa, 2009; Floysand, Barton y Román, 2010; Haarstadt y Campero, 2012; Instituto Nacional de Derechos Humanos 2012; Tricot, 2013; Miller, 2014).

El silencio de las regiones fue roto por actores que pusieron en la agenda reivindicaciones vinculadas a territorios específicos o contextos regionales amplios. Con ello la representación territorialmente homogénea que se suponía conformaba la pluralidad 
política del espacio público se vio cuestionada por la emergencia de sujetos que portaban una diversidad territorial. Este movimiento no solo puso en entredicho el centralismo en la organización del Estado (Delamaza, Cunill y Joignant, 2012) y en el sistema de distribución de riqueza (Shejtman y Berdegué, 2004) sino además puso en evidencia que la supuesta homogeneidad territorial sobre la que se basaba el pluralismo político, descansaba sobre una idea simplificada y estandarizada del "nosotros". Se multiplican los actores, en diferentes escalas. Así, la estructuración de muchos conflictos parece haber cambiado, desplazándose desde los actores del sistema político nacional, que tradicionalmente ejercían la mediación entre la sociedad y el Estado (Garretón, 1985) hacia los escenarios territoriales. Su procesamiento político también cambió, toda vez que la mediación de los partidos políticos parece haber perdido fuerza en todos los contextos, experimentando a la vez un descenso en la valoración y legitimidad ciudadana, al tiempo que se ha modificado el vínculo entre actores sociales y partidos y han aparecido nuevos actores de mediación, como las redes internacionalizadas (Bowen, Fábrega y Medel, 2012), los funcionarios públicos relacionados al activismo de la sociedad civil (Abers y Von Bülow, 2011), así como coaliciones y alianzas de actores (Birner, Sharma y Palaniswaly, 2006), entre otros.

De manera que la emergencia política de esta "subjetividad regional" no solo consiguió poner en la agenda temas vinculados a los territorios, sino además instaló la condición territorial como un factor de diferenciación de los sujetos en el espacio público y por tanto, definió nuevos lugares desde los cuales concurrir a la pluralidad. Este es justamente el punto en que la emergencia de esta subjetividad regional dialoga con nuestro trabajo, pues pone en evidencia que el territorio y la región son espacios de producción de una singularidad diferenciada que mantiene un acceso aún parcial al espacio público. Resta entonces determinar la magnitud o profundidad de esas singularidades, respecto de lo cual este trabajo da un primer paso, siendo necesario otros esfuerzos, de orden cualitativo o bien referido a otras escalas territoriales (Delamaza y Thayer, en prensa).

Esta emergencia de subjetividades territoriales nuevas se enmarca en un contexto más amplio de debilitamiento global de los mecanismos de representación política (Beck, 2001). Este proceso que ha sido denominado "individualización", (Beck, 2006; Beck y Beck-Gersheim, 2003), “desmodernización" (Touraine, 1998), o "desanclaje" (Giddens, 2001), apunta a poner en evidencia el agotamiento de la capacidad de las instituciones políticas tradicionales para mediar entre la experiencia de los sujetos y el espacio público. Con ello se habría abierto un horizonte de incertidumbre en el plano privado y de transformación de las condiciones para la vida pública y para el ejercicio del poder y el gobierno (Blanco y Gomá, 2002; Castells, 1998). La emergencia de una pluralidad más 
compleja asentada en subjetividades territoriales se enmarca así en un proceso mayor en que la capacidad de las instituciones políticas y sociales para representar e integrar a los sujetos en una existencia común, estaría en crisis. De esta manera la nueva diversidad territorial conlleva un desafío de reforma institucional, sin la cual la mayor complejidad del espacio público corre el riesgo de ser moldeada y reducida por instituciones concebidas para representar sujetos organizados en base a unos referentes superados.

\section{Importancia de la percepción política: una aproximación operacional}

Las encuestas de opinión política se han desarrollado significativamente desde los primeros estudios pioneros de fines de los años 50 hasta la actualidad. Desde fines de los años 80 se comenzó a aplicar encuestas de opinión de manera periódica, las que han pasado a ser un producto muy valorado para la toma de decisiones políticas. Así, encuestas como la del Centro de Estudios Públicos (CEP), la empresa Adimark y el Centro de Estudios de la Realidad Contemporánea (CERC) se vienen aplicando y publicando en series que ya tienen más de 20 años. A ellas se agregan otras que han ido ganando presencia en el medio nacional como las realizadas por el Programa de Naciones Unidas para el Desarrollo (PNUD) para elaborar sus Informes de Desarrollo Humano del país (a partir de 1996) y los Informes de Auditoría a la Democracia (a partir de 2008), y la de la Universidad Diego Portales (UDP), la única entidad netamente académica, que lo hace periódicamente, en el último quinquenio ${ }^{3}$. También se aplican en el país encuestas internacionales periódicas como el Latin American Public Opinion Project (LAPOP), el Latinobarómetro y la Encuesta Mundial de Valores, que podrían ser incorporadas de manera más detallada en análisis posteriores siempre y cuando sus preguntas cumpliesen los requisitos mencionados y que se tenga acceso a las bases de datos ${ }^{4}$. En el presente artículo utilizamos la versión 2011 de la Encuesta Latinobarómetro.

Un trabajo anterior permitió dimensionar el alcance que han tenido los estudios por la vía de encuestas. En ese caso se trató de una recopilación de los estudios disponibles sobre participación entre 1990 y 2009 (Foster, 2010). De acuerdo con ese trabajo en el período se aplicó un total de 137 encuestas que incluyeron el tema de participación, con un total de 1.512 preguntas sobre el tema. Sin embargo, para lo que aquí interesa,

3 También la Universidad Católica publica una Encuesta Bicentenario con frecuencia semestral, pero ésta prácticamente no toca temas de contingencia política como los que aquí se analizan.

4 La Fundación Futuro cuenta con un Banco de Encuestas Nacionales. Sin embargo, su utilidad para propósitos comparativos es limitada, puesto que sólo se cuenta con los informes y no con las bases de datos. 
aunque se evidencia que los estudios se han incrementado significativamente desde mediados de la década pasada, estos se aplican muy mayoritariamente a muestras en la región Metropolitana y grandes ciudades (38\%) o muestras nacionales (52\%) (Foster 2010, p. 18). De las encuestas recopiladas sólo tres de ellas desagregaban sus datos por regiones. Los esfuerzos desagregados por región son escasos en Chile: la Encuesta Más Voces (telefónica, en sólo seis regiones) (2004); la Encuesta de Estratificación Social del Proyecto Desigualdades (2009) y el Observatorio Regional SUBDERE (2009), no publicado; así como la Encuesta de Percepción de Calidad de Vida Urbana, del Instituto Nacional de Estadísticas y el Ministerio de Vivienda, aplicada en 103 comunas en 2007 y 2011 (Observatorio Urbano, 2014). Salvo este último, no identificamos ningún estudio de frecuencia periódica como el caso de las encuestas nacionales.

Las implicancias de lo anterior son variadas. En el campo de las políticas públicas una limitación ya diagnosticada se refiere a su diseño e implementación homogénea en todo el territorio nacional, sin consideración de las particularidades regionales y territoriales. Son diversos los elementos que confluyen para que ello ocurra, entre ellos el alto costo de producir información desagregada, por el incremento del tamaño de la muestra y la falta de prioridad política e incentivos institucionales para hacerlo en un Estado unitario y centralizado (Delamaza, Cunill y Joignant, 2012). Pero eso produce un efecto de base: no se sabe lo que se piensa y quiere en las regiones y territorios en relación a las políticas públicas, antes que nada, porque no se formulan preguntas a los actores ni a la ciudadanía, respecto de lo que piensan y quieren. Este déficit de información se subsidia con información agregada a nivel nacional y en función de los criterios de la política "nacional", tanto en las preguntas que se formulan como en el universo de respuestas.

Existe mayor disponibilidad de información desagregada respecto de datos económicos y sociales de tipo objetivo. Instrumentos como la Encuesta CASEN, por su parte, produce abundante información desagregada, pero no incluye variables de percepción política. Incluso un módulo sobre participación en organizaciones se incluyó durante algunas versiones de la encuesta y luego se omitió en las siguientes. Resulta evidente la disparidad en cuanto a disponibilidad de información regionalizada. Mientras se cuenta con importantes series de información económica y social, son escasos los estudios que incorporan las variables subjetivas y de práctica ciudadana y las correlacionan con los datos anteriores. Entre las escasas excepciones se debe mencionar dos esfuerzos promovidos por la SUBDERE a fines del decenio pasado: la Encuesta Nacional de Estratificación Social 2009, que forma parte del Proyecto Desigualdades de la Universidad de Chile (www.desigualdades.cl) y el frustrado proyecto de Observatorio Regional 2009, cuyos datos nunca se publicaron. El proyecto Desigualdades ha 
publicado algunos informes de evidente interés para nuestro objeto (Barozet, Espinoza, Holz y Sepúlveda, 2009; Espinoza y Rabí, 2009; Méndez, 2008). También el PNUD se ha aproximado a estos temas, especialmente a través del primer Informe de Desarrollo Humano en Chile (PNUD, 1996) y el Informe de Desarrollo Humano Rural (PNUD, 2008). Aunque lo identifica como una necesidad, no ha emprendido encuestas regionales o diferenciadas por territorios.

Por tanto la situación de falta de información es especialmente aguda en relación a los datos subjetivos, atribución de liderazgos y opinión política en general, etc. Vale decir aquello que podría constituir una "opinión pública" regional. Esta ausencia redunda eventualmente en una devaluación de las políticas, reduciendo su impacto e introduciendo distorsiones y desajustes en su implementación. Adicionalmente dificulta sensiblemente el surgimiento y visibilidad de una "masa crítica regional" que incida en los temas que le son propios e impulse el proceso descentralizador y de equidad territorial. Vale decir, la falta de información "invisibiliza" a las regiones y territorios frente a los decisores nacionales y, paradójicamente, frente a ellas mismas en el debate público (Delamaza, Cunill y Joignant, 2012).

Las constataciones hechas en los apartados anteriores son de carácter práctico. Sin embargo, nada indican respecto de si la desagregación por escalas territoriales involucra una diferencia empíricamente significativa en términos de la opinión ciudadana, puesto que no existe comparación entre los resultados de los estudios. Es necesario, por tanto, verificar el supuesto de que dicha diferencia existe y es relevante. Si los resultados comparados indican tal diferenciación, ello abre una perspectiva de investigación futura muy relevante. Pero ello debe ser demostrado, cumpliendo los requisitos metodológicos para la comparabilidad que es lo que pretende este trabajo en las áreas que aborda.

La técnica utilizada para permitir comparaciones válidas entre diferentes investigaciones cuantitativas sobre percepción política se basa en el llamado "meta-análisis". Esta técnica es utilizada para realizar una revisión y recopilación de la literatura de un conjunto de resultados empíricos con el propósito de integrar los hallazgos respecto a un tema. Normalmente a partir de esta técnica es posible generar una síntesis de la literatura científica disponible, sacando conclusiones de los distintos trabajos revisados. Asimismo, permite comparar y valorar la calidad de las distintas metodologías utilizadas (Hunter, 2004, p. 17). En este caso la utilizamos para comparar distintas encuestas de opinión, siempre y cuando se cumplan algunas condiciones metodológicas. 
Si bien es cierto que muchas veces se citan datos de encuestas diferentes para contrastar resultados, no siempre se revisa el proceso metodológico que hace posible las comparaciones y evita distorsiones o resultados ilusorios. En la presente comparación se utilizó sólo encuestas de tipo presencial, aplicadas a muestras aleatorias de representatividad proporcional, aplicadas en un período relativamente acotado de tiempo -entre 2011 y 2013- y que no coincidieran en las mismas fechas, puesto que ello conlleva el riesgo de que algunos encuestados se repitan (puesto que las muestras nacionales y regionales coinciden en algunos territorios). También se consideró un número limitado de preguntas, que fueron formuladas de la misma manera en las diferentes encuestas. Con ello nos referimos a la forma de preguntar así como a la forma de categorizar las respuestas, pues se trata en todos los casos de preguntas cerradas.

El meta-análisis se utiliza para integrar los resultados de distintos estudios, para revelar patrones simples de relaciones que subyacen a la literatura científica, proporcionando así una base para el desarrollo de ideas que son comunes, o bien identificar los efectos distorsionadores de error de muestreo, error de medición y otros artefactos que producen la ilusión de resultados contradictorios. Por último, una de las finalidades del meta-análisis es estimar la mayor precisión posible de la información sobre un tema, de modo de mejorar la calidad de los resultados (Hunter y Schmidt, 2004, p. 12). De este modo, en el presente artículo resulta pertinente utilizar esta técnica, ya que permite revisar, sistematizar e integrar los diferentes estudios sobre percepción política, logrando así una mayor precisión sobre los elementos que describen la investigación en el tema que interesa.

\section{Variables de la percepción y subjetividad política}

Para la selección de las variables a utilizar en la comparación de datos de encuestas, se consideró tanto elementos operacionales como conceptuales. Entre los primeros interesa contar con información comparada, por lo cual el criterio principal es la disponibilidad de los datos en el formato adecuado. Mientras mayor cantidad de estudios utilicen la misma pregunta y las mismas categorías de respuesta, mayor valor tiene la variable, desde el punto de vista estrictamente operacional.

En el terreno conceptual, por cierto, la percepción política es un campo vastísimo que requeriría de esfuerzos de investigación mucho más amplios que el que aquí se presenta. Como una manera de precisar el objeto optamos por privilegiar variables 
subjetivas que pueden considerarse centrales para un enfoque de gobernanza democrática (Blanco y Gomá, 2002; Mayntz, 2005). Este enfoque considera amplia la noción de gobierno, considerando como elemento central el involucramiento de la ciudadanía. Ello permite privilegiar las variables que aquí hemos seleccionado: democracia, confianza y satisfacción personal. Por otra parte, con un número discreto de variables se debe cubrir un espectro relativamente amplio de asuntos vinculados a la percepción política, de tal manera de contrastar si la diferenciación regional opera de distinto modo según el ámbito de percepción seleccionado.

Una primera dimensión seleccionada alude a la percepción tanto normativa como factual de la democracia y su funcionamiento en el país. Esta dimensión se ha ido consolidando en los estudios como adhesión personal a la democracia en relación a otros sistemas políticos. Esto constituye una percepción normativa de la democracia en general y en abstracto. De allí que sea necesario también combinar esto con la percepción que se tiene de su funcionamiento, aspecto en el cual hemos seleccionado el juicio sobre qué tan democrático considera el país, lo cual constituye un punto de vista aplicado por parte de los encuestados a la realidad percibida en el país.

Las variables anteriores se refieren a la democracia en un plano social y político general, es decir entrañan un juicio de percepción política sobre el "sistema democrático" en su conjunto. Es interesante complementar entonces dichos juicios con aquellos que involucran a la relación entre las propias personas en su relación con el sistema institucional. De allí que una segunda dimensión de percepción seleccionada se refiere a la confianza. La variable confianza ha sido incluida normalmente como un componente relevante del capital social en diferentes niveles (Durston, 2000; PNUD, 2000). Se considera relevante el vínculo que se establece con otras personas, así como con las instituciones con las cuales se interactúa. Al respecto caben, sin embargo, dos consideraciones conceptuales: en primer término los dos ámbitos de la confianza no pueden considerarse correlacionados, de hecho pueden evolucionar de manera diferenciada, pues responden a lógicas distintas. A pesar de ello, ambas son importantes para el objeto que nos ocupa. Una segunda cuestión es que la percepción de confianza puede tener un carácter "basal", es decir revelar una actitud o predisposición general (ser confiado o desconfiado frente a algo) o bien revelar una evaluación contingente, que alude más bien al funcionamiento de una institución en un momento determinado (PNUD, 2014). La confianza sería un componente inmaterial complejo del vínculo, que influiría en la predisposición de las personas hacia la participación, la adhesión a la democracia, etc. Por ello resulta una

5 Algunas encuestas preguntan respecto de la satisfacción personal con la democracia, pero en ese caso no tenemos la base de comparación adecuada con el Barómetro Regional. 
dimensión complementaria significativa de incluir. Empíricamente se ha constatado que los niveles de confianza en Chile, tanto interpersonal como en las instituciones de la democracia representativa son extremadamente bajos, lo cual, sin duda, otorga mayor interés a la comparación que proponemos, en la medida que confirme o refute en el ámbito regional dicha percepción.

Una tercera dimensión combina también el plano personal con el de la situación general del país y refiere a la satisfacción con la situación que se vive. En Chile contemporáneo se ha constatado una mejor valoración de la situación personal que de la valoración de la situación del país. En este sentido la "crítica social" emergería desde distintas fuentes, no siempre correlacionadas estrictamente con la insatisfacción con la propia vida. De hecho utilizaremos una clasificación que combina cuatro posibilidades de conformidad derivadas del cruce de la pregunta por satisfacción general con la vida y percepción de suficiencia de ingresos.

Tabla 1

Matriz de Conformidad

\begin{tabular}{lll}
\cline { 2 - 3 } & Satisfacción con la Vida & Insatisfacción con la Vida \\
\hline Percepción de Ingresos Suficientes & Conformes & Inconformistas \\
Percepción de Ingresos Insuficientes & Resignados & Disconformes \\
\hline
\end{tabular}

Fuente: Elaboración propia.

Vale decir que dichas variables operan de manera singular y en esa medida son comparables a escala regional, pero también lo hacen de manera combinada, estableciendo la tipología y su comportamiento a diferente escala.

Una vez establecidas las comparaciones de escala en las tres dimensiones seleccionadas, introducimos dos variables adicionales que pueden establecer patrones de diferenciación política con respecto a las percepciones. También en este caso queremos analizar la pertinencia e importancia de la escala regional. En otras palabras, ¿funcionan los diferenciadores políticos del mismo modo en ambas escalas territoriales? ¿Cómo se distribuyen las preferencias y las prácticas políticas según la escala territorial? ¿Hasta qué punto la transformación socio política de los territorios se expresa en estos elementos diferenciadores? Ello es especialmente relevante en contextos de fuertes transformacio- 
nes en el campo de las ideologías y de emergencia de movimientos sociopolíticos de base territorial. Para abordar esta dimensión de "diferenciación política" hemos elegido una combinación de variables que incluye la autoadscripción en los ejes convencionales de la política (derecha / izquierda) y una variable de "práctica social": la participación en organizaciones.

En síntesis, el diagrama conceptual y operativo que se propone para el análisis es el siguiente:

Tabla 2

Diagrama conceptual

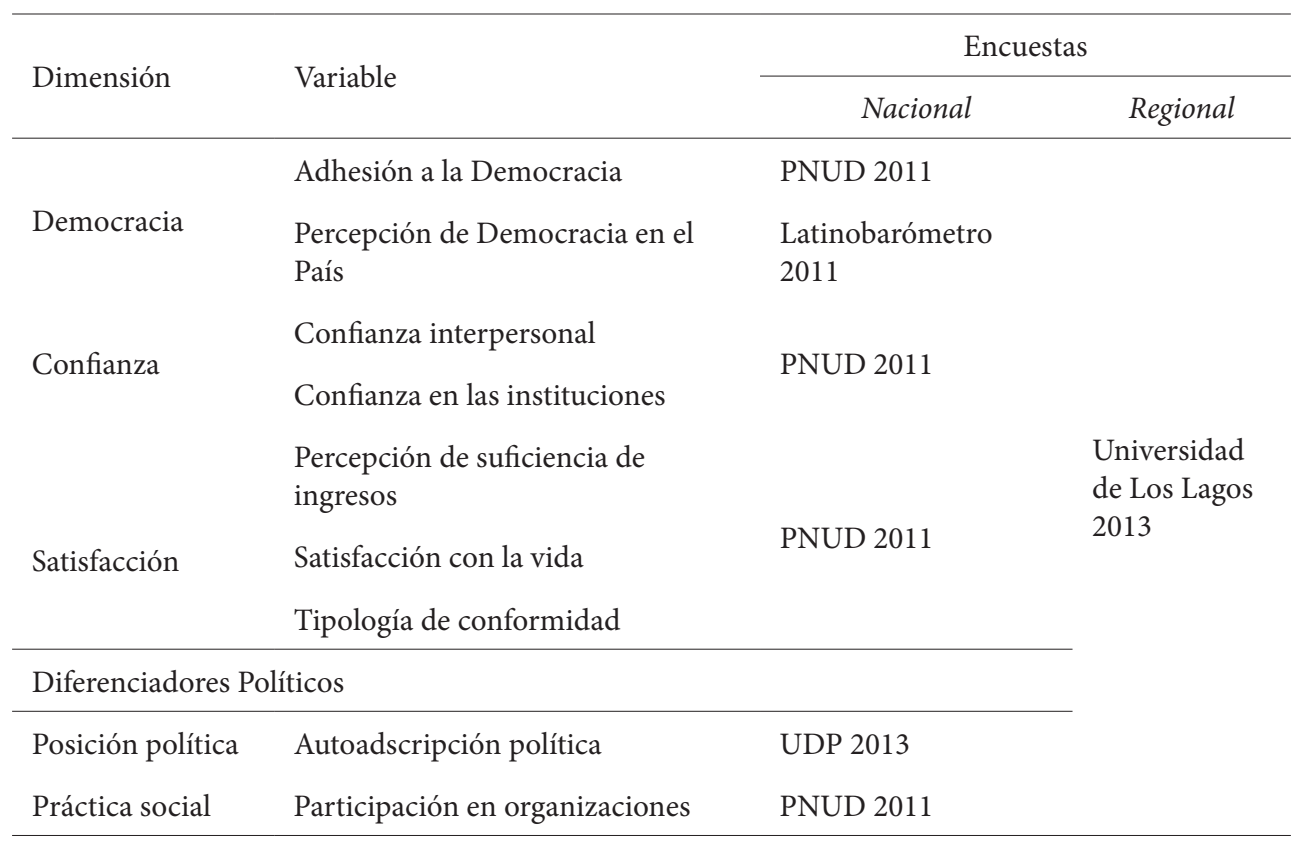

Fuente: Elaboración propia. 


\section{Resultados: la importancia del territorio}

En esta sección abordamos cada una de las dimensiones seleccionadas analizándolas según dos tipos de aproximaciones territoriales, relacionadas a la pluralidad de la configuración de la opinión política y la noción de un "nosotros" que la contenga. En primer término se compara las opiniones recogidas por encuestas nacionales a muestras representativas proporcionales de todo el país. Algo así como la "opinión promedio" del país, contrastado con la "opinión promedio" frente a la misma pregunta en tres contextos regionales diferentes. Para ello se revisaron las bases de datos de las encuestas y se construyó un dato estrictamente comparable entre ambas escalas, que se presenta en los gráficos siguientes. Un segundo nivel de análisis avanza más allá de la comparación promedio nacional respecto de promedio regional, para establecer comparaciones entre las regiones. Las diferencias que allí se puedan apreciar permitirían pensar que la escala territorial de cada una de las regiones define particularidades que es importante tomar en cuenta.

Para establecer la significación de las diferencias encontradas, luego de su presentación en términos porcentuales comparativos en los gráficos, se aplicaron pruebas de dos tipos: la de intervalos de confianza, considerando los errores muestrales de las encuestas nacionales y regionales; y pruebas de diferencias estadísticas al asumir que las encuestas nacionales y regionales son comparables bajo el supuesto de que ambas pueden ser consideradas "muestras independientes".

La primera permite establecer si las diferencias entre escalas territoriales superan los intervalos de confianza derivados de los márgenes de error de cada una de las encuestas o si existe un segmento que se superpone entre ambas, en términos del margen de error. Esto supondría que existe una alta probabilidad de que los parámetros poblacionales pudiesen coincidir y, con ello, no sería posible identificar una diferencia clara entre ambos parámetros.

La segunda, busca identificar si la diferencia que pudiese existir entre los estadísticos muestrales, tanto entre regiones como entre éstas y los estadísticos muestrales nacionales, difieren con algún grado de significancia estadística. Para ello, se aplicaron diferentes técnicas de comparación de diferencias, por un lado, pruebas de diferencias de medias como t-Student y ANOVA si las variables a comparar eran continuas y, también, pruebas de Chi-cuadrado y $\mathrm{Z}$ de proporciones en el caso de las variables discretas (más detalles en nota metodológica). 


\section{Adhesión a la democracia y su evaluación en el país}

Utilizando como base de comparación la encuesta del PNUD 2011 y el Barómetro Regional de la Universidad de Los Lagos 2013 (este último será la base de comparación de la escala regional para todos los casos), se tiene una primera aproximación a las diferencias. Si bien en todas las escalas la opción ampliamente mayoritaria es la que prefiere la democracia a cualquier otra forma de gobierno, el porcentaje oscila entre el 61,4\% (precisamente el porcentaje promedio nacional) y un 82,1\% en la región de Los Ríos, vale decir más de 20 puntos de diferencia. El patrón de adhesión/indiferencia democrática se refuerza si se considera que el porcentaje más alto que señala que "a la gente como uno le da lo mismo un gobierno democrático que uno autoritario" se ubica también en la escala nacional $(26,4 \%)$ y el menor en Los Ríos $(10,8 \%)$ como se aprecia en la figura 1 . Las otras dos regiones se ubican en el estrato intermedio: Bío Bío manifestando mayor adhesión y menor indiferencia y Los Lagos a la inversa, muy cerca de los promedios nacionales. La prueba de confianza de intervalo indica que existen diferencias de intervalo precisamente de las categorías extremas entre las dos regiones de mayor adhesión democrática con respecto al promedio nacional. Mientras que la significación estadística se verifica en todas las regiones en relación al parámetro nacional. ¿Qué explica esas diferencias? Eso es algo que no podemos saber con los actuales datos, pero sí sabemos que la diferencia de adhesión democrática es importante en magnitud y significativa estadísticamente. 


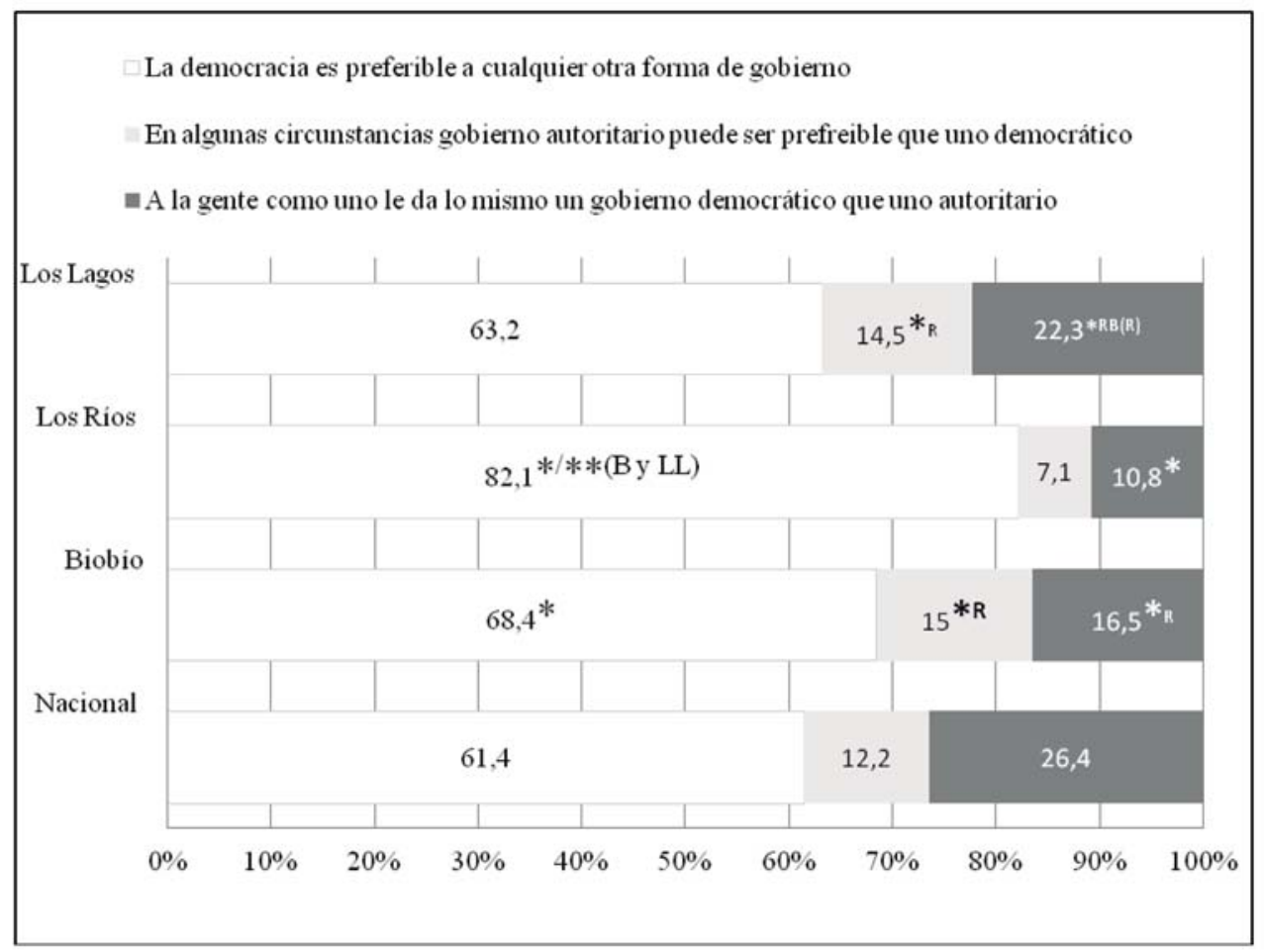

Figura 1: Nivel de adhesión a la democracia. Regiones y total nacional (\%). Existen algunas diferencias de intervalo en algunas categorías de las regiones de Bío bío y Los Ríos ${ }^{*}$ ) con el parámetro nacional. Y de Los Ríos respecto de las otras dos regiones $\left({ }^{*}\right)$. (b) Existen diferencias estadísticamente significativas de todas las regiones con el parámetro nacional $\left(\chi^{2} ; \mathrm{p}<0,05\right)$. (c) Existen diferencias estadísticamente significativas a favor las regiones de Biobío y Los Lagos respecto Los Ríos $\left({ }^{\star} \mathrm{R}\right)$ y de Los Lagos respecto de Biobío $\left({ }^{\star} B\right)$ en distintitas categorías $\left(\chi^{2} ; \mathrm{p}<0,05\right)$. Fuente: Elaboración propia (Encuesta PNUD 2011 y Barómetro Regional 2013).

El segundo parámetro elegido es una evaluación de la situación del país, asunto en el cual, en principio, podría esperarse una mayor incidencia de la escala regional. Tomando aquí la encuesta Latinobarómetro 2011 para la determinación del parámetro nacional, se verifica dicha diferenciación, puesto que este se ubica sustancialmente por encima de la evaluación de la democracia en el país que se tiene en las tres regiones consultadas. A diferencia de la variable de adhesión democrática, sin embargo, es la región de Los Ríos la que más se acerca evaluativamente al parámetro nacional. Ambas pruebas -confianza y significación- revelan diferencias para todas las regiones con el parámetro nacional, como se aprecia en la figura 2. 


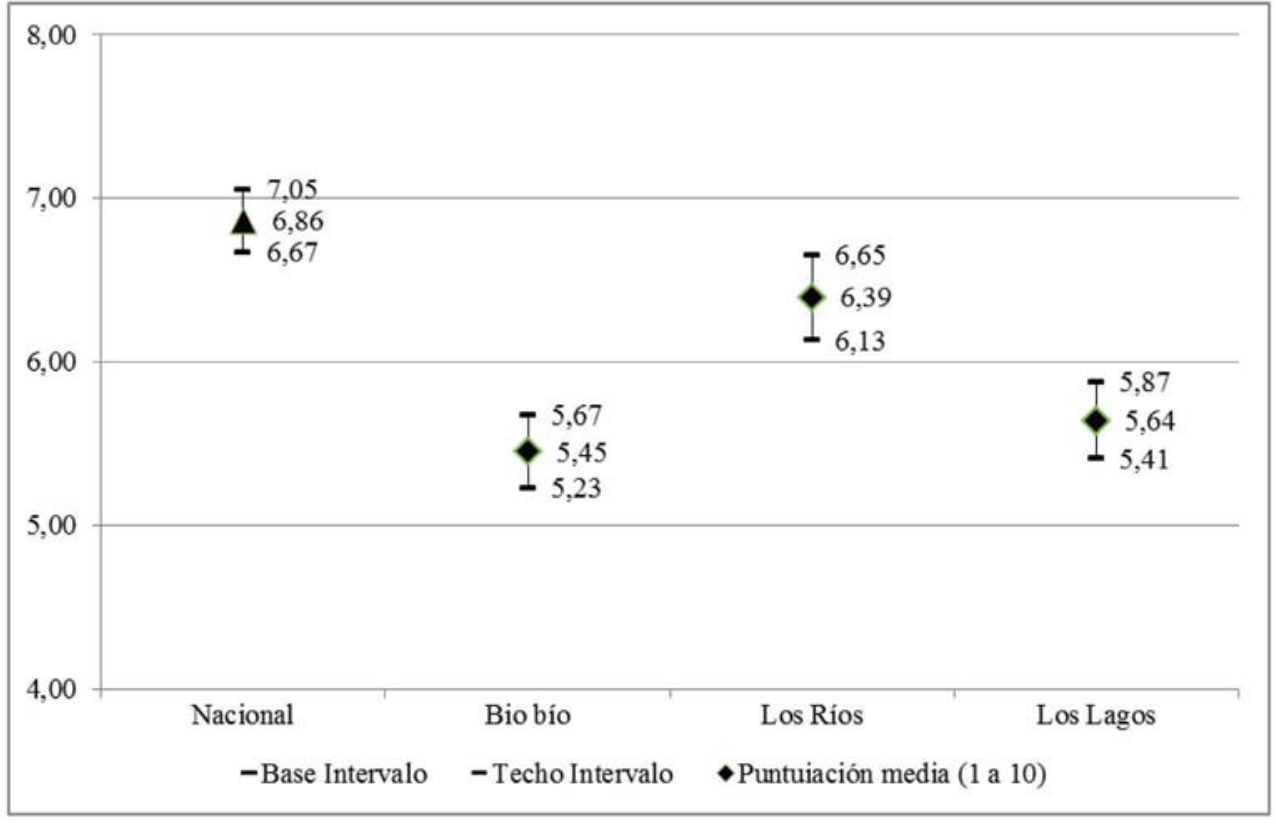

Figura 2: Percepción sobre el nivel de democracia en el país. Regiones y país. (Escala de 1 = nada democrático, $\mathbf{1 0}$ = totalmente democrático). (a) Existen diferencias de intervalo de todas las regiones con el parámetro nacional; (b) Existen diferencias estadísticamente significativas de todas las regiones con el parámetro nacional (t-Student; $\mathrm{p}<0,05)$; (c) Existen diferencias estadísticamente significativas y de intervalo de la nota media, particularmente a favor de Los Ríos ${ }^{* *}$ ) respecto de Biobío y Los Lagos (ANOVA; p<0,05). Fuente: Elaboración propia (Latinobarómetro 2011 y Barómetro regional 2013).

En términos de las diferencias entre las distintas regiones, el segundo nivel del pluralismo en la esfera pública, tenemos que existen diferencias estadísticamente significativas en adhesión a la democracia y en la nota media sobre evaluación democrática del país entre las regiones del Bío Bío y Los Lagos con respecto a Los Ríos. También Bío Bío y Los Lagos se diferencian de modo significativo en distintas categorías de la adhesión democrática (Figura 1). 


\section{Confianza}

¿Qué sucede con la confianza? Los datos que presentamos en las figuras 3 y 4 muestran el panorama de diferenciación regional en relación a esta variable. En cuanto a la confianza interpersonal, el parámetro nacional, recogido de la Encuesta PNUD 2011 resulta notoriamente superior a los de todas las demás regiones. La diferencia más acusada se aprecia con la región de Los Ríos, donde alcanza 15 puntos porcentuales. Ambas pruebas arrojan diferencias significativas para todas las regiones en relación al parámetro nacional. Adicionalmente se puede señalar que las encuestas regionalizadas indican una tendencia de la confianza que resulta contraintuitiva respecto de algunas explicaciones que asocian positivamente ruralidad y confianza. Es precisamente la región más rural la que presenta menores niveles de confianza, como se visualiza en la figura 3.

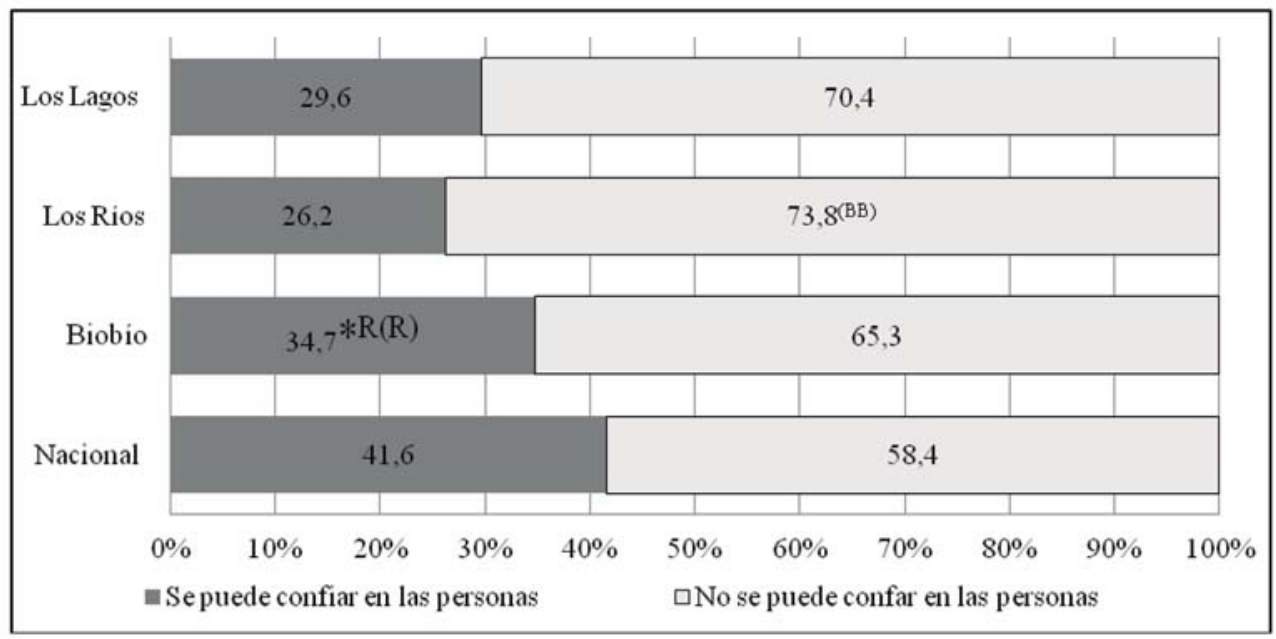

Figura 3: Confianza interpersonal. Regiones y país (\%). (a) Existen diferencias de intervalo de todas las regiones con el parámetro nacional; (b) Existen diferencias estadísticamente significativas de todas las regiones con el parámetro nacional $\left(\chi^{2} ; \mathrm{p}<0,05\right)$; (c) Existen diferencias estadísticamente significativas a favor de Biobío respecto de Los Ríos $\left({ }^{\star R}\right)$ para la opción «se puede confiar en las personas» ( $\chi^{2}$; p<0,05). Fuente: Elaboración propia (Encuesta PNUD 2011 y Barómetro regional 2013). 


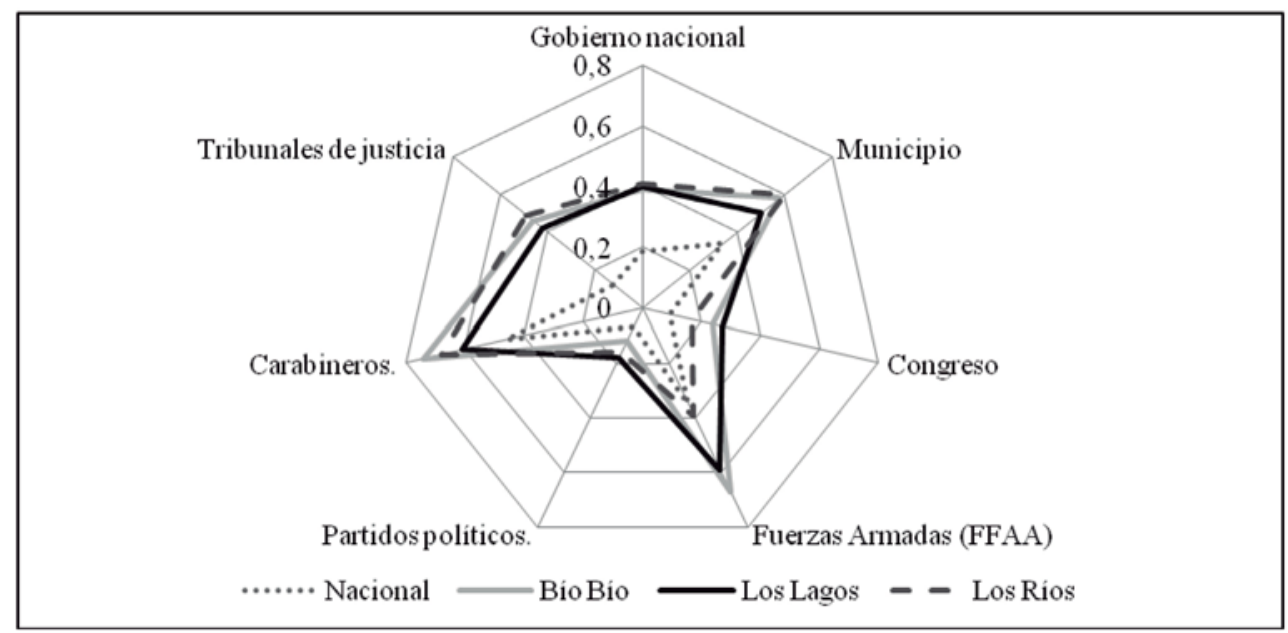

Figura 4: Confianza en las instituciones. Regiones y país (\% bastante + mucha confianza). (a) Existe diferencia de intervalo de todas las regiones e instituciones respecto del parámetro nacional, a excepción de partidos políticos en Bio Bío y FFAA en Los Ríos; (b) Existen diferencias estadísticamente significativas de todas las regiones con el parámetro nacional $\left(\chi^{2} ; \mathrm{p}<0,05\right)$; (c) Existen diferencias estadísticamente significativas para prueba de comparación bilateral $(Z)$ entre las tres regiones en todas las categorías de confianza institucional en todos los niveles de confianza. Destaca que Biobío y Los Lagos difieren a la par respecto de Los Ríos al comparar los distintos niveles de confianza para la mayoría de instituciones evaluadas. Fuente: Elaboración propia (Encuesta UDP 2013 y Barómetro regional 2013).

La confianza en las instituciones resulta una variable más compleja de medir, por cuanto se pregunta sobre diferentes instituciones, en las cuales se deposita mayores o menores grados de confianza, como se representa en la figura 4. Lo primero que llama la atención como tendencia general, es la menor confianza en las instituciones que manifiesta el parámetro nacional respecto de las tres regiones consideradas y en relación a todas las instituciones evaluadas. Esto demuestra el distinto comportamiento que tiene la confianza institucional respecto de la interpersonal, cuestión que sólo se visualiza cuando se pone el parámetro nacional en relación con otros, de otro modo resulta difícil interpretar las magnitudes de diferencia. El país tiene mayor confianza interpersonal que (al menos) tres de sus regiones y en cambio menor confianza en todas sus instituciones que la existente en sus regiones. En el extremo inferior, de la menor confianza, se encuentran los partidos políticos, tanto en el país como en sus regiones. Pero mientras en el promedio nacional le siguen en desconfianza a muy corto intervalo el Congreso y los tribunales de justicia, en las regiones la situación es muy diferente. Allí el Congreso recibe una mayor cuota de confianza y los tribunales se ubican en un rango todavía muy superior, similar a 
la que recibe el gobierno nacional. Este último, en el caso del promedio nacional, resulta mucho menos confiable. Las instituciones que reciben mayor confianza de la población resultan ser las fuerzas armadas, los carabineros y los municipios, en ese orden. En las tres instituciones la confianza regional es significativamente mayor que la nacional. La diferencia es estadísticamente significativa en todos los casos, así como lo es la de intervalo en la mayoría de ellos (Figura 4).

En cuanto a la muestra de confianza en siete instituciones seleccionadas para comparar con el parámetro nacional se tiene que hay diferencias significativas en todas ellas en relación a lo nacional y diferencias de intervalo en la mayoría de ellas. Entre las regiones, las diferencias siguen un patrón repetitivo que sitúa a las regiones de Bío Bío y Los Lagos de una parte y a Los Ríos de otra respecto de la mayor parte de las instituciones consultadas, al menos en alguna de las categorías de respuesta en cada una. Las particularidades locales se pueden deber a diversos factores, algunos relacionados simplemente con las características del territorio. A modo de ejemplo puede señalarse que la confianza en las fuerzas armadas puede estar relacionada con la inexistencia de regimiento militar en la región de Los Ríos, versus la importante presencia de algunas ramas de las fuerzas armadas en las otras dos regiones. Pero la reiteración del patrón, indica que ello puede asociarse también a condiciones que diferencian de manera importante a la región de Los Ríos: su mayor pobreza y ruralidad, su menor tamaño y su condición de región de reciente creación, entre otras. A su vez, las instituciones que reciben mayor confianza en las tres regiones estudiadas son parcialmente las mismas: carabineros en las tres, las fuerzas armadas y universidades (Los Ríos y Los Lagos). Pero sobre todo el perfil de cada región se expresa en las instituciones que reciben alta confianza pero no coinciden entre las regiones: iglesias en Bío Bío y organizaciones sociales en Los Ríos (Barómetro Regional, 2013).

\section{Percepción de ingresos suficientes y satisfacción con la vida}

Esta dimensión se expresa en dos variables que, combinadas, permiten definir una tipología de cuatro posiciones, relativas a la conformidad, tal como se expresó previamente. En cuanto a percepción de ingresos suficientes los promedios regionales son sensiblemente mayores al nacional: mientras los primeros oscilan entre 57,3\% en Los Ríos (la región más pobre y objetivamente con menores ingresos) y 52,3\% en Bío Bío, el promedio nacional se ubica sólo en un 49,8\%. La variable satisfacción con la vida se comporta de otro modo. Allí el promedio nacional (62\%) está ubicado entre el 73,7\% de Bío Bío y el 58\% de Los Ríos. 
Al formularse la combinación de las variables como tipología (Figuras 5, 6, 7 y 8 ) se evidencian diferencias estadísticamente significativas de todas las regiones con el parámetro nacional en al menos en dos categorías: más satisfechos y menos inconformistas y disconformes en Bío Bío; más inconformistas y menos resignados en Los Ríos; más satisfechos y menos disconformes en Los Lagos. Se expresa, por lo tanto una interesante diversidad regional en relación a la tipología.

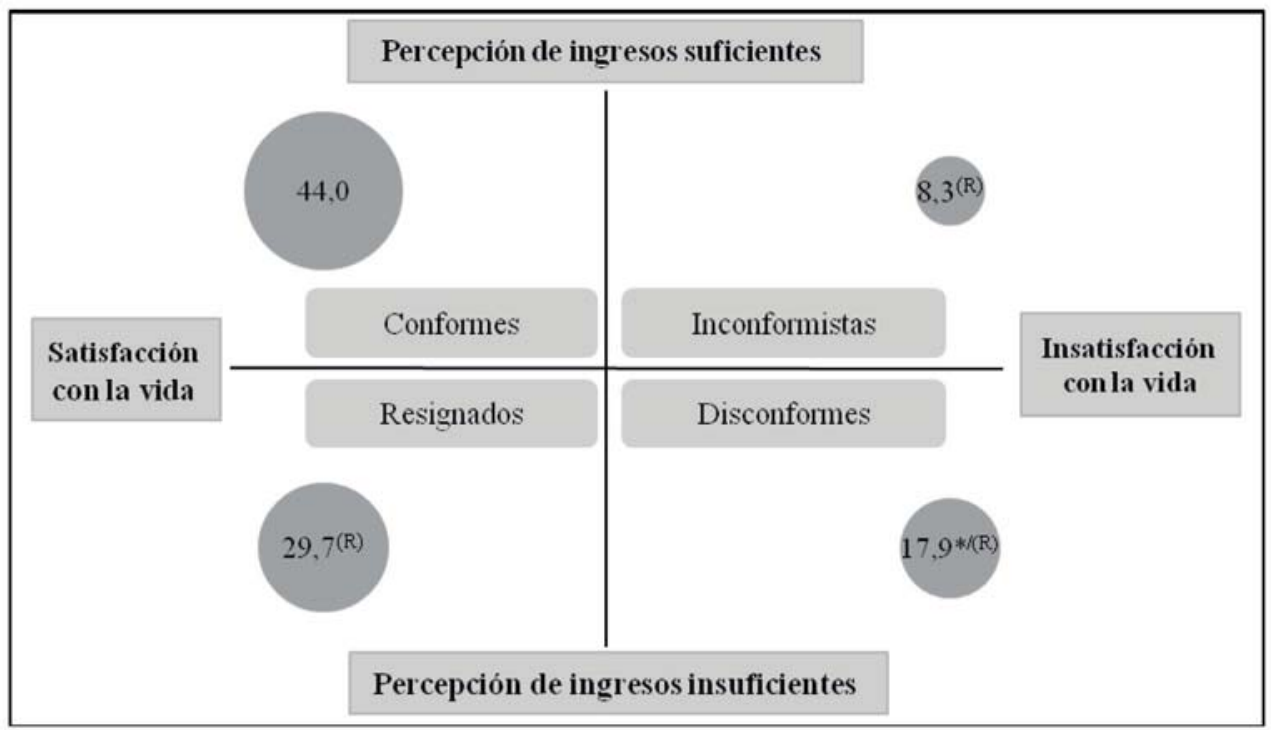

Figura 5: Satisfacción general con la vida y percepción de suficiencia de ingresos. Bío Bío (\%). (a) Diferencia de intervalo con el parámetro nacional ${ }^{\star} /^{(\mathrm{R})}$ Diferencia de intervalo entre la región del Bío Bío y Los Ríos; (b) Existen diferencias estadísticamente significativas a favor Biobío en la categoría «resignados» respecto de Los Lagos y Los Ríos $\left(\chi^{2} ; \mathrm{p}<0,05\right) ;(\mathrm{c})$ Existen diferencias estadísticamente significativas a favor de Biobío para las categorías «conformes» $\mathrm{y}$ «resignados» respecto de las proporciones nacionales. Y a favor de esta última en «Inconformes» $\mathrm{y}$ «disconformes» $\left(\chi^{2} ; \mathrm{p}<0,05\right)$. Fuente: Elaboración propia (Regional Barómetro 2013). 


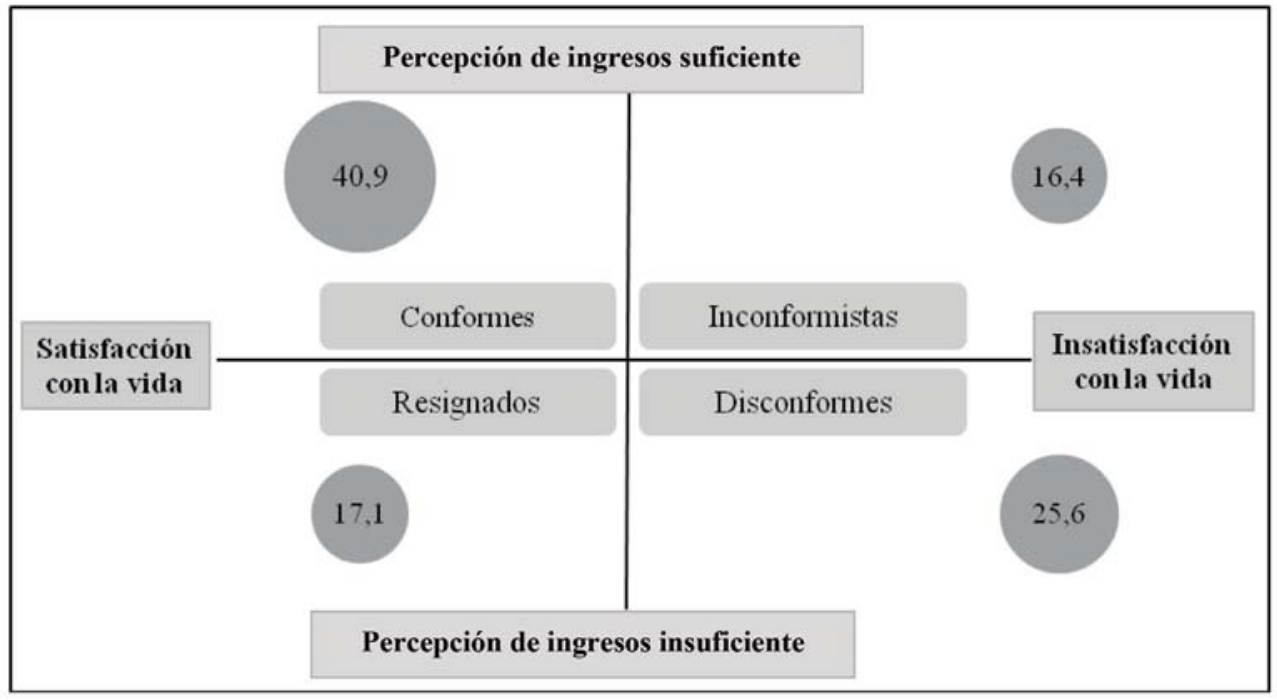

Figura 6: Satisfacción general con la vida y percepción de suficiencia de ingresos. Los Ríos (\%). (a) Existen diferencias estadísticamente significativas a favor de Los Ríos en las categorías «inconformistas» $\mathrm{y}$ «disconformes» respecto de Bío Bío $\left(\chi^{2} ; \mathrm{p}<0,05\right)$; (b) Existen diferencias estadísticamente significativas a favor Los Ríos la categoría «inconformista» respecto de las proporciones nacionales. Y a favor de esta última en «resignados» $\left(\chi^{2} ; \mathrm{p}<0,05\right)$. Fuente: Elaboración propia (Regional Barómetro 2013). 


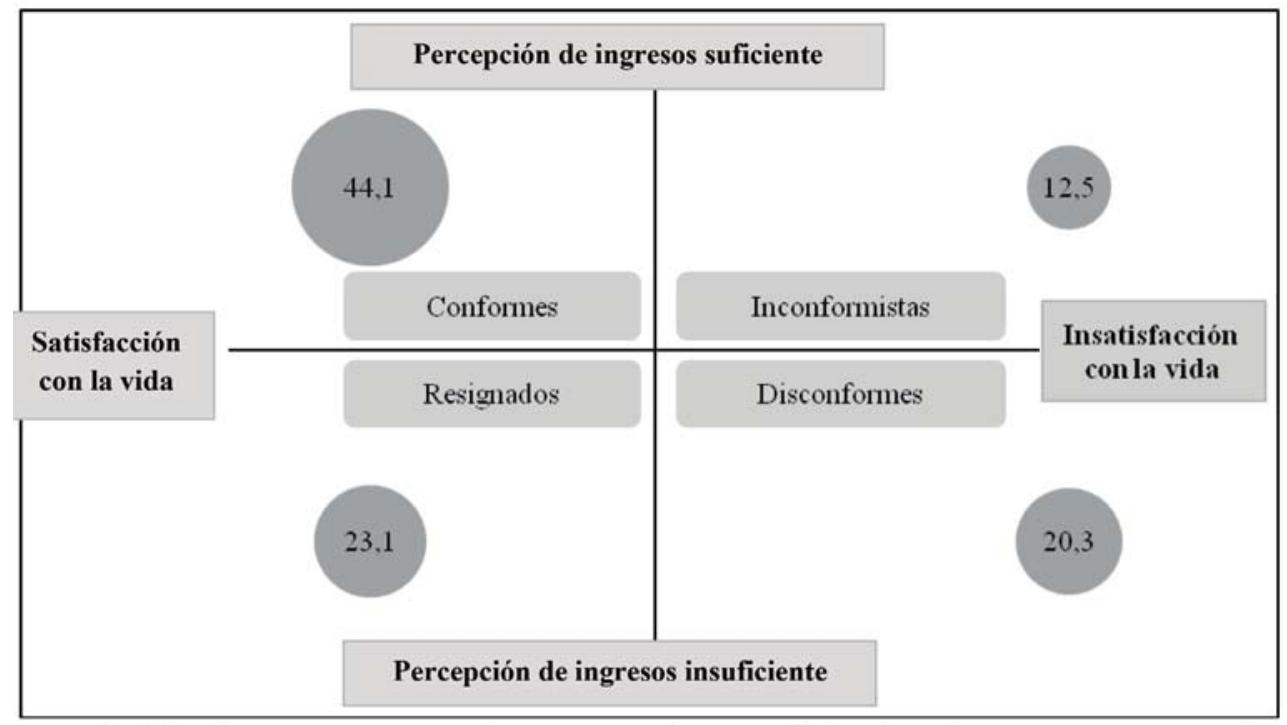

Figura 7: Satisfacción general con la vida y percepción de suficiencia de ingresos. Los Lagos (\%). (a) Existen diferencias estadísticamente significativas a favor de Los Lagos en la categoría «resignados» respecto de Los Ríos ( $\left.\chi^{2} ; \mathrm{p}<0,05\right)$; (b) Existen diferencias estadísticamente significativas a favor de Los Lagos para la categoría «conformes» respecto de las proporciones nacionales. Y a favor de esta última en «disconformes» $\left(\chi^{2} ; \mathrm{p}<0,05\right)$. Fuente: Elaboración propia (Regional Barómetro 2013). 


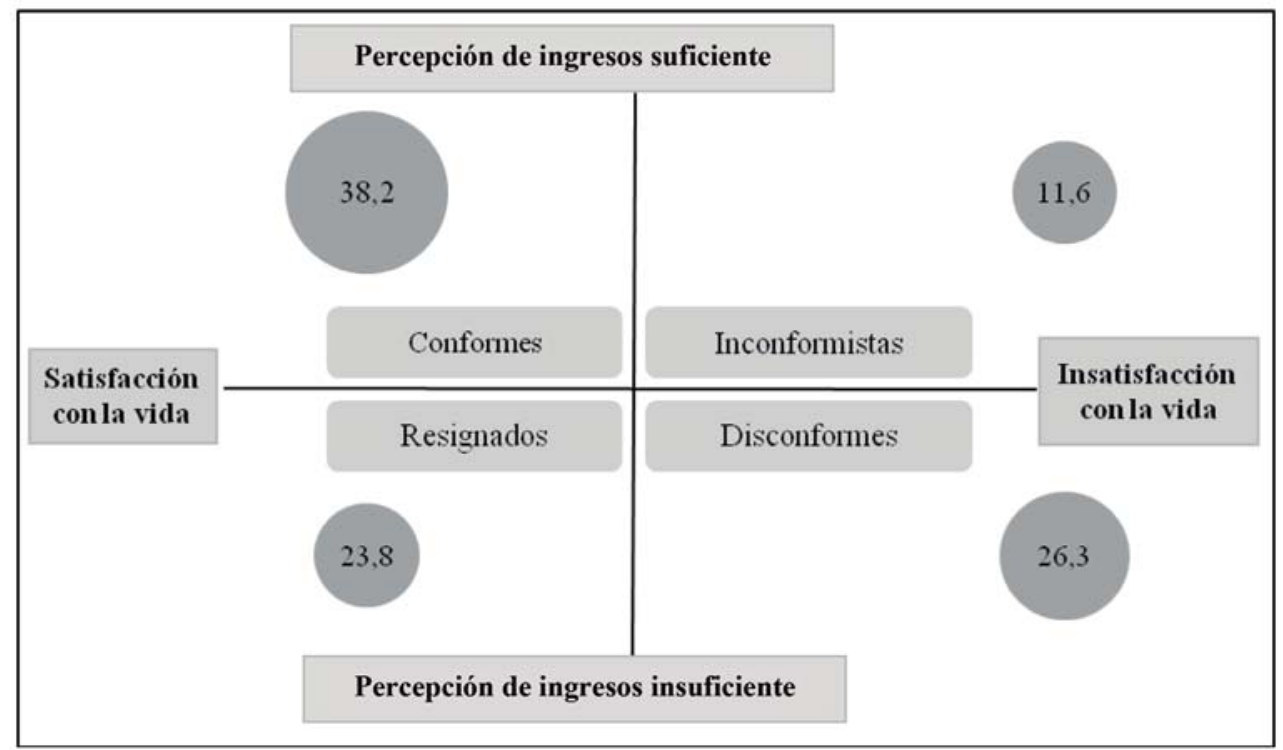

Figura 8: Satisfacción general con la vida y percepción de suficiencia de ingresos. País (\%). (a) Existe diferencia de intervalo entre la categoría "disconformes" en Bío Bío y el parámetro nacional; (b) Existen diferencias estadísticamente significativas de todas las regiones con el parámetro nacional en, al menos, una categoría (detalladas en láminas regionales). Fuente: Elaboración propia (Latinobarómetro 2011).

\section{Variables de diferenciación política: autoadscripción política y participación}

Si abordamos un clásico diferenciador político, como es la autoadscripción en el eje derecha / izquierda, vemos que también se pueden verificar diferencias interesantes al comparar escalas territoriales, como se aprecia en la figura 9. Estas, sin embargo son válidas tanto para los intervalos como para la significación estadística sólo entre las regiones de Los Ríos y Los Lagos, por una parte y el promedio nacional por la otra, para la puntuación media obtenida en el eje derecha / izquierda. No ocurre lo mismo en la región de Bío Bío. Entre las regiones, la diferencia significativa se produce en Los Ríos, que aparece significativamente más “izquierdista” que el promedio nacional, seguido por Los Lagos, mientras Bío Bío se ubica más a la derecha y más cerca del promedio nacional. 


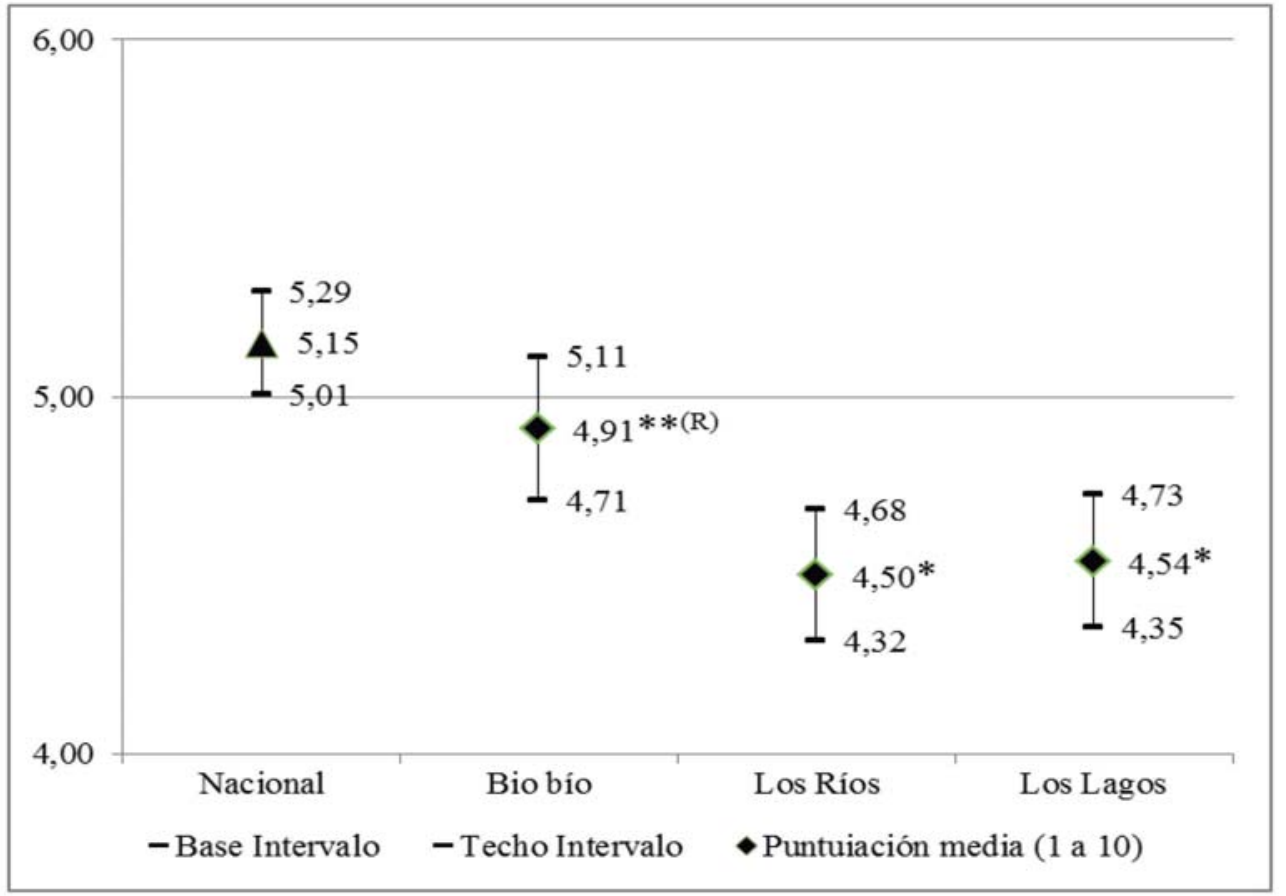

Figura 9: Autoadscripción política. Regiones y país. (Escala $1=$ izquierda $10=$ derecha). (a) Existe diferencia de intervalo entre la puntuación media de Los Ríos y Los Lagos $\left(^{*}\right)$ y el parámetro nacional; (b) Existen diferencias estadísticamente significativas solo entre las medias de Los Lagos y Los Ríos $\left(^{*}\right.$ ) y el parámetro nacional, (t-Student $\mathrm{p}<0,05$ ); (c) Existen diferencias estadísticamente significativas de la nota media, a favor de Los Ríos IV ${ }^{* *}$ ) respecto de las Biobío y Los Lagos (ANOVA; $<<0,05$ ). Y de Intervalo de Bío Bío respecto de Los Ríos. Fuente: Elaboración propia (Encuesta UDP 2013 y Barómetro regional 2013).

En cuanto a la participación en organizaciones, una variable que reporta prácticas, el promedio nacional es más alto que los regionales, siendo el menor de todos el de Los Ríos. Aplicadas las pruebas, todas las regiones indican diferencias tanto de intervalo como estadísticamente significativas con el parámetro nacional. Nuevamente es la región de Los Ríos la que muestra diferencias estadísticamente significativas con respecto a las regiones de Bío Bío y Los Lagos. 


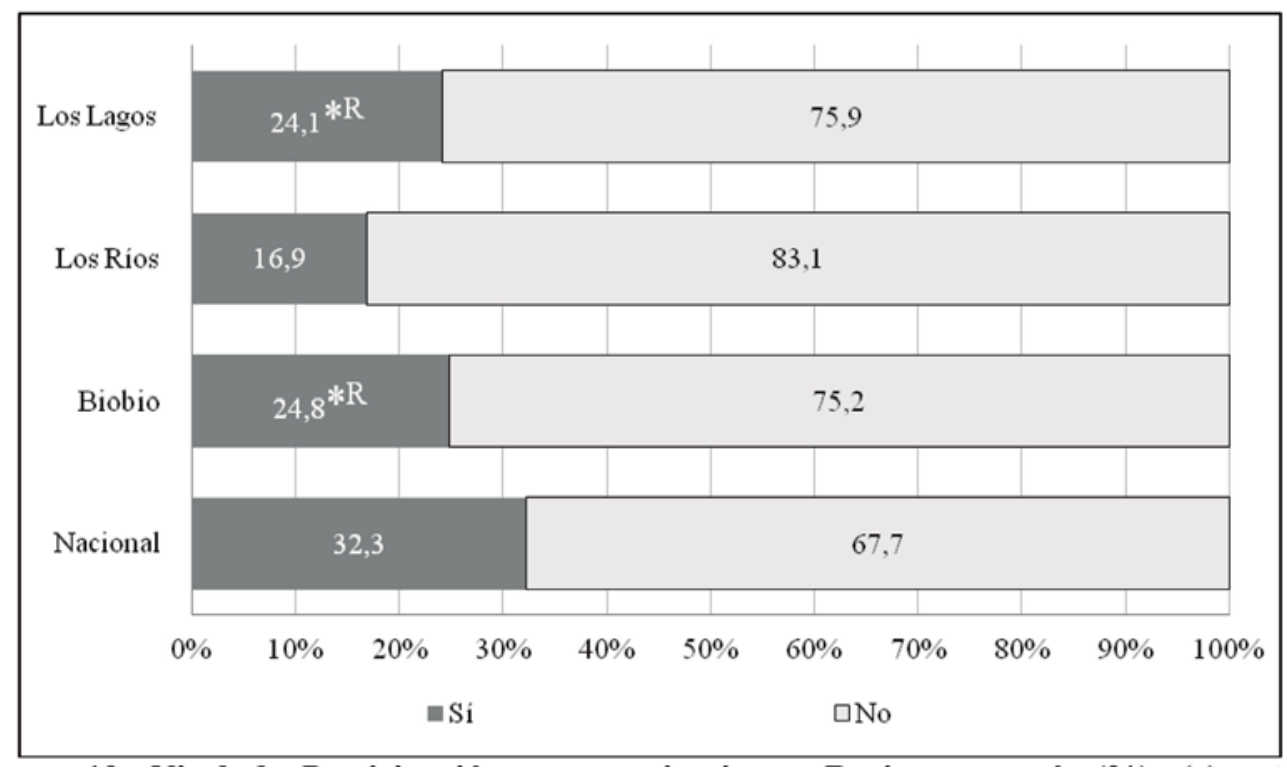

Figura 10: Nivel de Participación en organizaciones. Regiones y país (\%). (a) Existen diferencias de intervalo de todas las regiones con el parámetro Nacional; (b) Existen diferencias estadísticamente significativas de todas las regiones con el parámetro Nacional $\left(\chi^{2} ; \mathrm{p}<0,05\right)$; (c) Existen diferencias estadísticamente significativas a favor las regiones VIII y X respecto de la XIV $\left({ }^{* R}\right)$ para la opción «participa» $\left(\chi^{2} ; \mathrm{p}<0,05\right)$. Fuente: Elaboración propia (Encuesta PNUD 2011 y Barómetro regional 2013).

\section{Conclusiones}

La búsqueda de elementos diferenciadores al interior de una sociedad nacional cobra nuevas significaciones ante el debilitamiento de los mecanismos tradicionales de integración y mediación política organizados precisamente en función del ámbito nacional y el correspondiente rol del Estado. La introducción de la variable territorial, que aparece plausible de acuerdo a diferentes dinámicas sociales y políticas, se revela a la luz de la información empírica como un diferenciador muy significativo. Esta constatación abre interesantes perspectivas de investigación, toda vez que puede extenderse a otros campos temáticos, donde la heterogeneidad puede ser mayor o menor. También resulta interesante introducir estudios cualitativos que permitan profundizar en aspectos más específicos de las diferencias encontradas utilizando muestras representativas de encues- 
tas de opinión. Por último, se abre también una línea de trabajo en cuanto a comparar los datos subjetivos con otros datos socioeconómicos de las regiones. Todo ello permitiría avanzar en determinar causas o patrones de regularidad en la diferenciación regional en el contexto nacional.

El trabajo presentado permite concluir que los promedios nacionales en relación a las variables de subjetividad política contienen en su interior altas tasas de desviación y por lo tanto sintetizan situaciones que presentan importantes diferencias entre sí. Conceptualmente ello obliga a realzar el valor del pluralismo en la constitución de la esfera pública y los sujetos que la conforman, incluyendo aquí a los originados en las regiones, con sus diferencias, un elemento débilmente considerado en la tradición centralista y unitaria de la política chilena. Reafirma este aserto el hecho que las regiones estudiadas no corresponden a regiones extremas, las que muy posiblemente presentarían aún mayores niveles de diferenciación con respecto al promedio. ¿Cuáles son los territorios que "equilibran" lo aquí presentado para conformar el promedio nacional? No lo sabemos: puede ser un efecto debido a las regiones del norte del país, en la medida que aquí se consideró solo regiones del centro-sur. O bien producto del peso relativo que puede tener la región Metropolitana en cualquier muestra nacional ${ }^{6}$. $\mathrm{O}$ a las regiones extremas del país. También allí surge una línea de investigación de alta productividad: se debe ampliar la cantidad de territorios a los cuales se interrogue para conocer su perfil propio.

Pero no se trata tan sólo de que el promedio esconde diferencias, sino también que dichas diferencias no responden a un patrón único. Vale decir funcionan en direcciones diferentes según sea el problema o la variable que se considere, lo cual ayuda a configurar perfiles específicos de cada territorio, en este caso, de nivel regional. Así sucede cuando se considera, por ejemplo, la tipología relativa a la conformidad, en una región como Los Ríos: mientras la percepción de ingresos contraría lo que podría pensarse al respecto, los habitantes de la región manifiestan mucho menor satisfacción con la vida, incrementándose el número de los inconformistas en relación a las otras muestras y los disconformes en relación a las otras dos regiones. En esta última categoría Los Ríos se parece al promedio del país. Desde un punto de vista de la gobernanza democrática, la consideración de la subjetividad es clave para la determinación de las metas y estrategias de política pública. En este caso se concluye que dicha subjetividad puede diferir sensiblemente de lo que externamente pudiera determinarse.

6 La base de elaboración de las muestras nacionales es el conjunto de la población, lo cual, traducido a regiones, le otorga un peso mucho mayor a las regiones más densamente pobladas, como la región Metropolitana de Santiago, volviendo estadísticamente menos visibles a las menos pobladas. 
También la investigación avaló la idea de que las regiones son diferentes entre sí, aún aquellas contiguas o cercanas, puesto que la muestra no se construyó por oposiciones radicales entre los diferentes casos. Esto hace que deba considerarse en los estudios no solo la diferenciación entre Santiago y regiones, como tiende a suceder en la mirada centralista. También se requiere considerar cada territorio en su singularidad. En este trabajo, las diferencias entre Los Ríos y las otras dos regiones se mostraron altamente significativas, tanto como las que existen con el promedio nacional. Metodológicamente esto lleva a considerar las escalas territoriales como un factor de mayor relevancia, puesto que el estudio muestra indicios de que la escala determina en parte los resultados. $\mathrm{Si}$ consideramos que hasta 2007 la región de Los Lagos incluía también a la actual Los Ríos, podemos suponer fácilmente que las diferencias internas en la región no se advertían, salvo que se introdujesen muestras de menor tamaño como aquí se ha hecho. Sin ello la visión del perfil de la región era muy diferente. De acuerdo a los objetivos de investigación, entonces, es que debe establecerse las escalas territoriales más adecuadas para la construcción de muestras.

Todas las variables elegidas muestran diferenciación significativa tanto entre regiones y el país como entre las propias regiones, esa es la conclusión fuerte del trabajo. En cuanto a los ámbitos temáticos relativos a la gobernanza democrática sobre los cuales se consultó, resalta en especial la pauta de diferenciación entre las tres regiones y el país, respaldada por la estadística, en relación a la confianza, particularmente por el hecho que la confianza en las instituciones aparece mucho mayor en las regiones, mientras lo contrario ocurre con la existente entre las personas. También debe destacarse la particular mayor diferenciación de la región de Los Ríos, pauta que se verifica de modo sistemático en relación a todas las variables.

La constitución de un "nosotros" susceptible de expresarse en una esfera pública democrática supone la inclusión de una diversidad de sujetos. Los territorios están siendo una fuente del surgimiento de dichos sujetos sociales provistos de relevantes perfiles propios en el ámbito político. Dichas realidades deberán ser consideradas en la visión que tenemos del país, así como en las propuestas políticas. 


\section{Referencias}

Abers, R. y Bülow, M. Von (2011). Movimentos sociais na teoria e na prática: como estudar o ativismo a través da fronteira entre estado e sociedade? Sociologias, 13 (28).

Angell, A., Lowden, P. y Thorp R. (2001). Decentralizing Development: The Political Economy of Institutional Change in Colombia and Chile. Oxford: Oxford University Press.

Arendt, H. (2003). La condición humana. Buenos Aires, Argentina: Paidos.

Barozet. E., Espinoza, V., Holz, R. y Sepúlveda, D. (2009). Estratificación social en regiones: ¿qué oportunidades ofrecen las regiones en Chile? Santiago, Chile: Proyecto Desigualdades.

Barómetro Regional (2013). Encuesta Realizada por el Centro de Investigación Sociedad y Políticas Públicas, CISPO - ULAGOS. Disponible en http://cispo.ulagos.cl/

Beck, U. (2006). La sociedad del riesgo. Hacia una nueva modernidad. Barcelona, España: Paidós.

Beck, U. y Beck-Gersheim, E. (2003). La individualización. El individualismo institucionalizado y sus consecuencias sociales. Barcelona, España: Paidós.

Bengoa, J. (2009). El conflicto mapuche en Chile: indígenas, institucionalidad y relaciones interétnicas. En J. López y M. Gutiérrez (Coords.), América indígena ante el siglo XXI (pp. 109-142). Madrid, España: Siglo XXI.

Birner, R., Sharma, N. y Palaniswaly, N. (2006). The political economy of electricity supply to agriculture in Andra Pradesh and Punjab. Washington D.C., Estados Unidos: IFPRI.

Blanco, I. y Gomá, R. (eds.) (2002). Gobiernos locales y redes participativas. Barcelona, España: Ariel.

Boisier, S. (2000). Chile: la vocación regionalista del régimen militar. EURE, 26 (77), 81-107.

———. (2010). Territorio, Estado y Sociedad en Chile. Santiago, Chile: Mago Editores.

Bowen, S., Fábrega, F. y Medel, R. (2012). Movimientos sociales rurales y problemática medioambiental: la disputa por la territorialidad. Psicoperspectivas, 11 (1), 204-225. 
Castells, M. (1998). ¿Hacia el Estado Red? Ponencia presentada en el Seminario Sociedad y reforma del estado, organizado por el Ministerio de Administracao Federal e Reforma Do Estado, República Federativa do Brasil. Sao Paulo, pp. 26-28.

Delamaza, G. (2014). Enhancing Democracy: citizen participation and public policies in Chile. Oxford: Berghahn Books.

Delamaza, G., Cunill, N. y Joignant, A. (2012). Nueva agenda de descentralización en Chile. Sentando más actores a la mesa. Santiago, Chile: Universidad de Los Lagos, RIL Editores.

Delamaza, G. y Thayer, E. (en prensa) Percepciones políticas y prácticas de participación como instrumento para la gobernanza de los territorios. Un análisis comparado de escalas territoriales en la macrorregión sur de Chile. EURE.

Durston, J. (2000). ¿Qué es el capital social comunitario? Santiago, Chile: CEPAL.

Espinoza, V. y Rabí, V. (2009). Capital social y civismo en las regiones chilenas. Proyecto Desigualdades, Santiago de Chile.

Falleti, T. (2010). Decentralization and subnational politics in Latin America. Cambridge: Cambridge University Press.

Floysand, A., Barton, J. y Román, A. (2010). La doble jerarquía del desarrollo económico y gobierno local en Chile: el caso de la salmonicultura y los municipios chilotes. EURE, 36 (108), 123-148.

Foster, B. (2010). Estudio sobre el estado del arte de la investigación sobre participación ciudadana en Chile. Disponible en www.cispo.ulagos.cl

Gaete, E. (2012). La descentralización desde Santiago y Valparaíso. Revisión de tres proyectos de ley sobre descentralización en Chile. En G. Delamaza, N. Cunill y A. Joignant, Nueva agenda de descentralización en Chile. Sentando mas actores a la mesa (pp. 605 - 635). Santiago, Chile: Universidad de Los Lagos, RIL Editores.

Garretón, M. (1985). La problemática de la transición a la democracia en Chile. Una síntesis. Washington D.C., Estados Unidos: Kellog Institute.

Giddens, A. (2001). Consecuencias de la modernidad. Madrid, España: Alianza. 
Góngora, M. (1980) Ensayo histórico sobre la noción de Estado en Chile siglos XIX y XX. Santiago, Chile: Ediciones La Ciudad.

Haarstad, H. y Campero, C. (2012). Extraction, regional integration, and the enudring problema of local political spaces. En H. Haarstad (ed.), New political spaces in latin american natural resource governance (pp. 83-106). Estados Unidos: Palgrave Macmillan.

Hunter, J. y Schmidt, F. (2004). Methods of Meta-Analysis. Estados Unidos: Sage Publications, Inc.

Instituto Nacional de Derechos Humanos INDH (2012). Mapa de conflictos socio ambientales en Chile. Recuperado de http://www.indh.cl/mapa-de-conflictos-socioambientales-en-chile.

Joignant, A. (2012). ¿Descentralización functional o territorial? Elementos de sociología de los agentes políticos en tres regiones de Chile (1989 - 2009). En G. Delamaza, N. Cunill, N. y A. Joignant, Nueva agenda de descentralización en Chile. Sentando mas actores a la mesa (pp. 511 - 558). Santiago, Chile: Universidad de Los Lagos, RIL Editores.

Latinobarómetro (2011). Encuesta aplicada por la Corporación Latinobarómetro.

Lechner, N. (1988). Los patios interiores de la democracia. Subjetividad y democracia. Santiago, Chile: Flacso.

Mayntz, R. (2005). Nuevos Desafíos de la Teoría de la Gobernanza. En A. Cerrillo, La Gobernanza hoy: 10 Textos de Referencia (pp. 83 - 98). Barcelona, España: IIG, INAP.

Méndez, M. (2008). Proceso de descentralización e identidad regional: ¿cómo se perciben los habitantes de regiones y como se perciben los procesos de desarrollo regional? Proyecto Desigualdades, Santiago, Chile.

Miller, T. (2014). La Frontera. Forests and ecological conflict in Chile's frontier territory. Durham and Lindon: Duke University Press.

Navarrete, B. (2012). ¿Partidos regionales o partidos con inscripción regional? (1989 - 2009). En G. Delamaza, N. Cunill y A. Joignant, Nueva agenda de descentralización en Chile. Sentando mas actores a la mesa (pp. 559 - 584). Santiago, Chile: Universidad de Los Lagos, RIL Editores. 
Observatorio Urbano (2014). Recuperado de http://www.observatoriourbano.cl/index.asp

Programa de Naciones Unidas para el Desarrollo PNUD (1996). Informe sobre desarrollo humano en Chile. Santiago, Chile: PNUD.

-_-_. (2000). Mas sociedad para gobernar el futuro. Informe de Desarrollo Humano en Chile. Santiago, Chile: PNUD.

_-_- (2008). Desarrollo humano en Chile rural. Seis millones por nuevos caminos. Santiago, Chile: PNUD.

_- — . (2011). Encuesta aplicada por el Programa de Naciones Unidas para el Desarrollo.

-_- (2014). Auditoría a la democracia. Más y mejor democracia para un Chile inclusivo. Santiago, Chile: PNUD.

Schejtman, A. y Berdegué, J. (2004). Desarrollo territorial rural. Debates y Temas Rurales 1. Santiago, Chile: Rimisp- Centro Latinoamericano para el Desarrollo Rural.

Salazar, G. y Pinto, J. (1999). Historia contemporánea de Chile. Tomo I: Estado, legitimidad, ciudadanía. Santiago, Chile: LOM Editores.

Tricot, T. (2013). Autonomía. El movimiento mapuche de resistencia. Santiago, Chile: Ceibo.

Touraine, A. (1998) ¿Podremos vivir juntos? Iguales y diferentes. México D.F., México: Fondo de Cultura Económica. 


\section{Anexo}

\section{Nota metodológica}

Para el análisis regional, se usó la encuesta Barómetro Regional 2013 aplicada por el Centro de Investigación Sociedad y Políticas Publicas de la Universidad de Los Lagos. Esta se compone de tres muestras probabilísticas y representativas de las regiones de Bío Bío, Los Lagos y Los Ríos, con errores muestrales de 4,1 puntos porcentuales. Para el análisis nacional se usaron: la encuesta de Desarrollo Humano en Chile 2011, el Latinobarómetro 2011 y la encuesta nacional de opinión pública de la UDP de 2013; todas ellas con márgenes de error entre los 2 y 2,8 puntos porcentuales. Para estimar las posibles diferencias entre los datos regionales y nacionales, se exploraron dos técnicas que permitieron la comparación.

Primero, se compararon los intervalos de confianza para la estimación de parámetros poblacionales según los estimadores muestrales y márgenes de error que presenta cada encuesta y siempre considerando las correcciones y ponderaciones definidas en los apartados metodológicos que cada una de las mediciones ha especificado. Segundo, se realizó la comparación de las encuestas como si estas fuesen muestra independientes, considerando, tanto que los periodos de levantamiento de la información fueron distintos en cada una de las mediciones, como que las preguntas coincidentes permitín asumir dicha comparativa. Dependiendo de la naturaleza de las variables, esto es, si eran discretas o continuas, se aplicaron test de Chi-cuadrado y comparación de proporciones (Prueba z) para las primeras, y pruebas de T-Student y ANOVA para las segundas. Ello permiió aproximarse a las diferencias entre las encuestas considerando un criterio de significancia estadística, complementario del anterior y, por tanto, discriminar las diferencias meramente nominales de aquellas que en su distribución presentan patrones particulares y con una alta probabilidad de no responder a efectos azarosos. 